\title{
Measurement of matric flux potential: a simple procedure for the hydraulic characterization of soils
}

\author{
H. F. M. ten Berge ${ }^{1}$, K. Metselaar ${ }^{2}$ and L. Stroosnijder ${ }^{2 *}$ \\ ${ }^{1}$ Centre for Agrobiological Research, P.O. Box 14, NL 6700 AA Wageningen, \\ Netherlands \\ ${ }^{2}$ Department of Soil Science and Plant Nutrition, Wageningen Agricultural Uni- \\ versity, P.O. Box 8005, NL 6700 EC Wageningen, Netherlands
}

Received 24 April 1987; accepted 18 May 1987

Key words: matric flux potential, measurement, steady state, soil water diffusivity, two-parameter rational function

\begin{abstract}
A new, simple and non-destructive method is proposed to measure directly soil matric flux potential as a function of water content. Soil water diffusivity is then found by differentiation of the measured curve. The steady-state method is based on a two-parameter expression for matric flux potential and requires few observations, obtained by standard equipment. Specific advantages are the method's applicability to the hydraulic characterisation of thin, brittle or hard samples. Experimental examples are presented and a theoretical analysis of errors due to gravity and nonstationarity is included. Where interest is in the integral characteristic matric flux potential, direct assessment of this function is expected to be more accurate than integration of a measured diffusivity curve. The method requires little labour but is relatively slow.
\end{abstract}

\section{Introduction}

The idea of combining a transport coefficient with a force-field potential into a flux potential was first introduced by Kirchhoff (1894), in the context of non-linear heat flow problems. This concept was adopted in the description of soil water transport by Klute (1952). The new flux potential was called 'matric flux potential' (Raats, 1970) to indicate that the associated gradient vector field is the flux field as governed by matric forces only. Although traditionally we are mainly dealing with potentials in force fields, it is fully legitimate to define potentials for any lamellar vec-

\footnotetext{
* Present address: Netherlands Universities Foundation for International Cooperation (NUFFIC) at Brawaijaya University, Graduate School, Soil \& Water Management Program, c/o Kotak Pos 176, Malang, Indonesia.
} 
tor field, such as the matric flux field considered here (Truesdell \& Toupin, 1960).

The use of the matric flux potential concept has been connected with the formulation of analytical solutions of the flow equation through the choice of an exponential relation for $k(p)$, the hydraulic conductivity as a function of soil water pressure $p$ (Gardner, 1958). Especially steady, multidimensional flows have been solved by combining the formulation in terms of matric flux potential with the exponential dependence of $k$ upon $p$ (e.g. Philip, 1971; Warrick, 1974; Raats, 1977).

Shaykewich \& Stroosnijder (1977) applied the concept of matric flux potential in the numerical simulation of water flow in soils. They indicated several advantages of matric flux potential over the conventional formulation which typically evaluates the transport coefficient and the driving force for the matric flux separately. Among these advantages are the avoidance of numerical overshoot, especially in the simulation of situations involving strong gradients (e.g. Vauclin et al., 1979) and the reduction of computation time. Finally, as a soil characteristic the matric flux potential curve is more accessible to interpretation in terms of fluxes than the soil water diffusivity or hydraulic conductivity function.

The integrated nature of a flux potential offers unique possibilities to the measurement of soil hydraulic properties, since at steady state the course of such a potential along the space coordinate in one-dimensional flow is known irrespective of the gradients in moisture content and pressure. This contribution proposes a very simple measurement procedure to assess directly the curve of matric flux potential $\Phi$ versus moisture content $\theta$ for undisturbed core samples. Since differentiation of $\Phi(\theta)$ with respect to $\theta$ yields the soil water diffusivity $D(\theta)$, the method described here may be viewed as an alternative to existing methods for the determination of $D(\theta)$.

\section{Theory}

The Kirchhoff transformation of the flux density equation implies substitution of the product $k \mathrm{~d} p$ by the differential $\mathrm{d} \Phi$. For the case of one-dimensional vertical flow with the space coordinate $z$ positive upward, we may then write the flux density $q$ as:

$$
q=-\frac{\mathrm{d} \Phi}{\mathrm{d} z}-k
$$

If we assign a pressure $p_{0}$ or a moisture content $\theta_{0}$ to a chosen reference condition, the matric flux potential at any pressure or moisture content may be defined with respect to this reference state as

$$
\Phi \equiv-\int_{p}^{p_{0}} k(p) \mathrm{d} p=-\int_{\theta}^{\theta_{0}} D(\theta) \mathrm{d} \theta
$$

provided the diffusivity be finite over the relevant $\theta$-domain. Raats and Gardner (1971) indicated that for realistic $k(p)$ functions this integral should converge. This was indeed found also on the basis of experimental data. Ten Berge (1986) ana- 


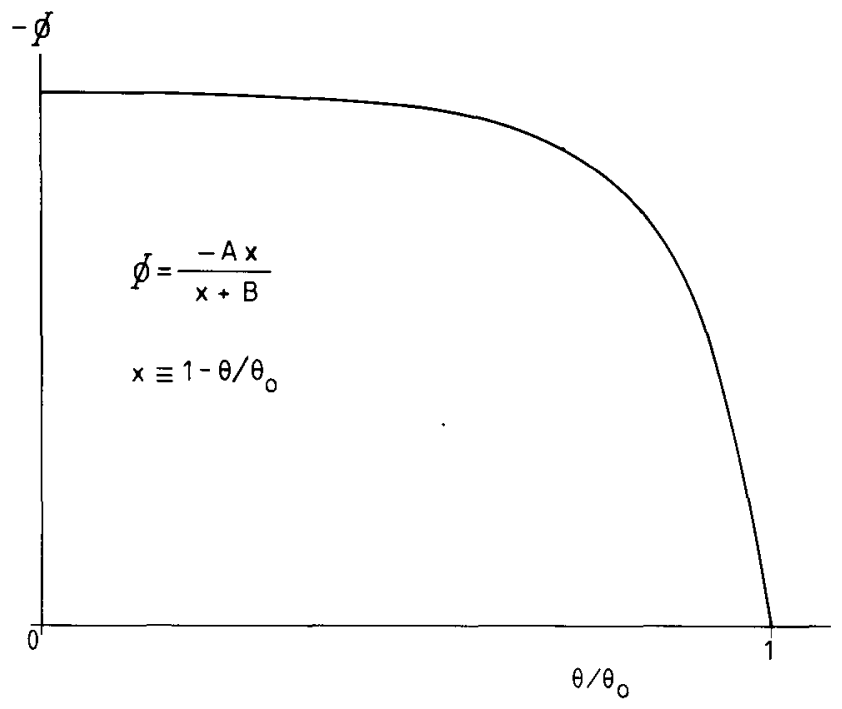

Fig. 1. Characteristic shape of the matric flux potential curve.

Table 1. Hydraulic properties of soils, based on data listed by Mualem \& Dagan (1976). $A$ and $B$ values were obtained after numerical integration of reported $D(\theta)$ curves, with moisture content at saturation as the reference value $\theta_{0}$.

\begin{tabular}{|c|c|c|c|}
\hline Soil & $\begin{array}{l}k_{\mathrm{s}} \\
10^{-2} \mathrm{~m} / \mathrm{d}\end{array}$ & $\begin{array}{l}A \\
10^{-2} \mathrm{~m}^{2} / \mathrm{d}\end{array}$ & $B$ \\
\hline Rideau clay & 29.6 & 5.52 & 0.0899 \\
\hline Yolo light clay & 0.86 & 0.32 & 0.1250 \\
\hline Buffalo silty clay & 6.82 & 0.45 & 0.0088 \\
\hline Glendale clay loam & 94.2 & 4.41 & 0.0965 \\
\hline Avondale loam & 24.0 & 5.55 & 0.0402 \\
\hline Guelph loam & 26.3 & 15.7 & 0.0595 \\
\hline Gilat loam & 18.7 & 6.56 & 0.0208 \\
\hline Grenville silt loam & 5.53 & 4.29 & 0.0242 \\
\hline Columbia silt loam & 5.05 & 2.73 & 0.0736 \\
\hline Ida silt loam & 7.97 & 3.07 & 0.0248 \\
\hline Mont Cenis silt loam & 1.22 & 1.30 & 0.1670 \\
\hline Yolo fine sandy loam & 1.28 & 1.73 & 0.0726 \\
\hline Vernal sandy loam & 19.1 & 4.20 & 0.1100 \\
\hline Bet Dagan I loamy sand & 552 & 141 & 0.0404 \\
\hline Panoche soil & 38.3 & 113 & 0.0230 \\
\hline Gravelly sand G.E.-9 & 23.7 & 11.8 & 0.0450 \\
\hline Hygiene sandstone & 108 & 107 & 0.0090 \\
\hline River sand & 193 & 33.2 & 0.0135 \\
\hline Sable $\mathbf{S} 2$ & 354 & 4.07 & 0.0046 \\
\hline Lakefield fine sand 3 & 457 & 125 & 0.0328 \\
\hline Lakefield fine sand 1 & 401 & 105 & 0.0254 \\
\hline Uplands sand & 158 & 18.7 & 0.0605 \\
\hline Nahal Sanai sand & 298 & 95.4 & 0.0182 \\
\hline
\end{tabular}


lysed $\Phi(\theta)$ relations as calculated from $k(\theta)$ and $p(\theta)$ data listed by Mualem \& Dagan (1976) for a wide variety of soils. All soils showed a characteristic shape of the $\Phi(\theta)$ curve (Fig. 1), which appears to be well described by a simple rational expression, making use of a scale factor $A\left(\mathrm{~L}^{2} \mathrm{~T}^{-1}\right)$ and a dimensionless shape factor $B$ :

$$
\Phi(\theta)=\frac{-A x}{x+B}
$$

with

$$
x \equiv 1-\theta / \theta_{0}
$$

Table 1 shows $A$ and $B$ values calculated for some soils from reported $k(\theta)$ and $p(\theta)$ data given by Mualem \& Dagan. It may be noted that differentiation of Eq. 3 directly gives a diffusivity function of the form used by Knight \& Philip (1974): $D(\theta)=a(b-\theta)^{-2}$, where $a$ and $b$ are constants.

The proposed experimental procedure makes use of two relations based on Eq. 3 that may be combined to yield the parameters $A$ and $B$. For a soil core of finite length $L$, the initial and boundary conditions required to obtain our two relations are:

$$
\begin{array}{llll}
\theta=\theta_{0} & \mathrm{t}=0 & 0 \leqslant z \leqslant L & \\
\theta=\theta_{0} & t \geqslant 0 & z=0 & \text { (bottom) } \\
\theta=0 & t>t_{\mathrm{c}} & z=L & \text { (surface) }
\end{array}
$$

where $t_{\mathrm{c}}$ is a critical time required to approach sufficiently near to a steady state. When gravity is neglected, both the flux at steady state and the total water loss that accompanies the shift from the initial to the final (steady) state, can be expressed in terms of $A, B$ and $\theta_{0}$. Since both the final flux and the total water loss can be assessed experimentally in a simple and non-destructive manner, the adoption of the above assumptions becomes very attractive. The flux density at steady flow is expressed as

$$
q=-\frac{\mathrm{d} \Phi}{\mathrm{d} z}=\frac{1}{L}\left\{\frac{A\left(1-\theta(L) / \theta_{0}\right)}{B+1-\theta(L) / \theta_{0}}\right\}
$$

which specializes under the conditions of Eqs. $5 \mathrm{~b}$ and $5 \mathrm{c}$ to give the maximum attainable steady flux through a soil column of length $L$ :

$$
q_{\max }=\frac{A}{(1+B) L}
$$

Clearly, this flux is only met when the demand at $z=L$, i.e. the imposed potential evaporation rate, surpasses a critical minimum value equal to $q_{\max }$. The profile of matric flux potential $\Phi(z)$ then follows from integration of Eq. 1, again neglecting gravity: 


$$
\Phi(z)=-q_{\max } z=\frac{-A z}{(1+B) L}
$$

Combination of Eq. 3 and Eq. 8 yields the 'complementary moisture content' profile:

$$
x(z)=\frac{z B}{(1+B) L-z}
$$

with $x$ defined as in Eq. 4. The total amount of water lost from the soil column during the transition from initial to final state is then found directly as a function of the shape factor $B$ by integration of Eq. 9:

$$
\int_{0}^{L} x(z) \mathrm{d} z=-B L\left\{(1+B) \ln \left(\frac{B}{1+B}\right)+1\right\} \equiv L \mathrm{f}(B)
$$

or in terms of total mass change $\Delta W$ :

$$
\Delta W=V \varrho_{1} \theta_{0} B\left\{(1+B) \ln \left(\frac{B}{1+B}\right)+1\right\}
$$

where $V$ and $\varrho_{1}$ are the sample volume and the specific density of water, respectively. With measured $\Delta W$ and $\theta_{0}$, Eq. 11 is solved for $B$ by iteration, for instance by a Newton-Raphson procedure. The scale factor $A$ is then obtained subsequently from Eq. 7 with the measured steady-flux value, $q_{\max }$.

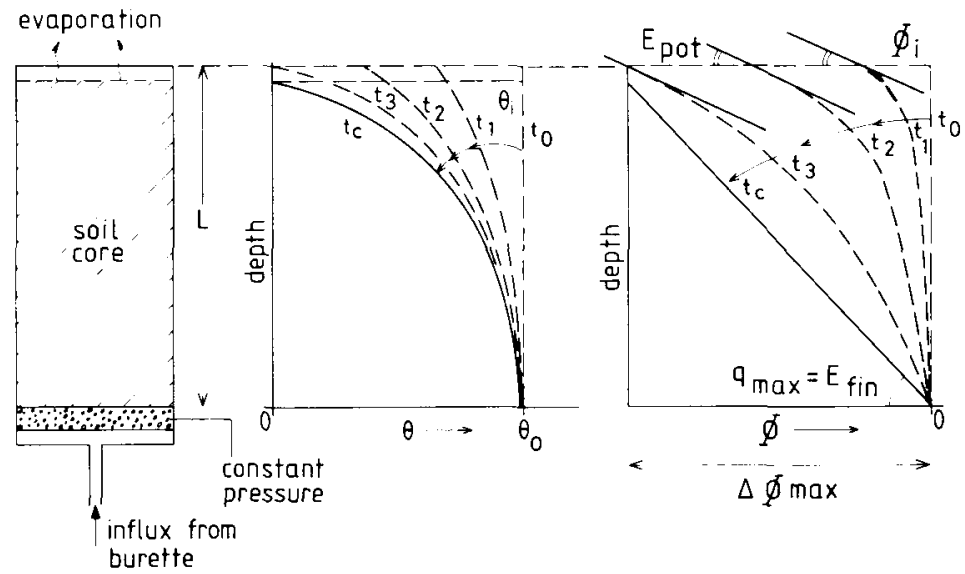

Fig. 2. Experimental set-up and graphical representation of $\theta(z)$ and $\Phi(z)$ at various time values. $E_{\text {fin }}$ is the final stationary evaporation rate. 


\section{Experimental procedure}

Fig. 2 illustrates the experimental set-up. The soil sample is connected to a water reservoir (burette) through a porous plate or cloth, in which the water pressure is maintained at the reference value $p_{0}$ by a mariotte arrangement. Initially the soil surface is covered and $\Phi(z)$ and $\theta(\mathrm{z})$ are supposedly constant throughout the sample, as indicated graphically in Fig. 2 . The initial sample weight is measured.

After removal of the surface cover, an upward flux is induced by evaporation, enhanced by the use of a hair-dryer. At first, evaporation is constant and $d \Phi / d z$ at the surface is fixed, being equal to the evaporative demand. As the surface dries beyond some limit (i.e. the moisture content where the relative humidity of soil air drops below 1), evaporation decreases. An essential condition is that the evaporative demand is larger than the maximum steady-state flux that the sample can support according to Eq. 7. Gradually the slopes $\mathrm{d} \Phi / \mathrm{d} z$ at the surface and at the bottom of the sample will approach the final value associated with the stationary flux $q_{\max }$. When the bottom flux as read from the burette becomes constant, the final weight of the core sample and the oven-dry weight are determined, thus completing the measurements required to assess the parameters $A$ and $B$ through Eqs. 7 and 11 .

\section{Some experimental examples}

The above procedure was applied to three soil materials: a very fine sand (packed), a sandy loam (packed), and a humous coarse sand (undisturbed field samples). Acrylate rings of $50 \mathrm{~mm}$ length and diameter were used. Values of $p_{0}$ were chosen at $-8,-5$ and $-5 \mathrm{kPa}$ for the three soils, respectively.

The measured $\Phi(\theta)$ curves according to Eqs. 7 and 11 are shown in Fig. 3. There appears to be some variation among the individual samples within one texture group, possibly associated with differences in dry bulk density for the field samples (humous coarse sand) and with packing irregularities in the artificial samples (very fine sand and sandy loam). The graphs clearly reflect the differences in shape factor $\mathrm{B}$ and scale factor A between the three soils.

As a check on the procedure, samples were mechanically sliced after determination of the final weight in order to assess independently the $\theta(z)$ profile by gravimetry. Combination of this profile with $\Phi(z)$ as obtained from $\Phi=-q z$ allows for a comparison between the two resulting $\Phi(\theta)$ curves: the one based on gravimetric sampling, the other based on Eqs. 3, 7 and 11. Fig. 4 compares one of the curves for each soil to $\Phi(\theta)$ as obtained by sectioning. Some inconsistency as to the shapes of the curves found by the two methods is observed. An inflection point in the $\Phi(\theta)$ curve would indicate a maximum in $D(\theta)$. The supposed relation in Eq. 3 is not in accordance with the occurrence of such a maximum. It seems likely, however, that here the observed $S$-shape reflected by the data points is due to redistribution during gravimetric sampling, because the discrepancy between the curves and data points occurs in the same manner for all three soils. Also, all analysed literature data (Table 1) support the validity of Eq. 3. The problem of redistribution during sampling was addressed extensively by van Grinsven et al. (1985) in their discussion 


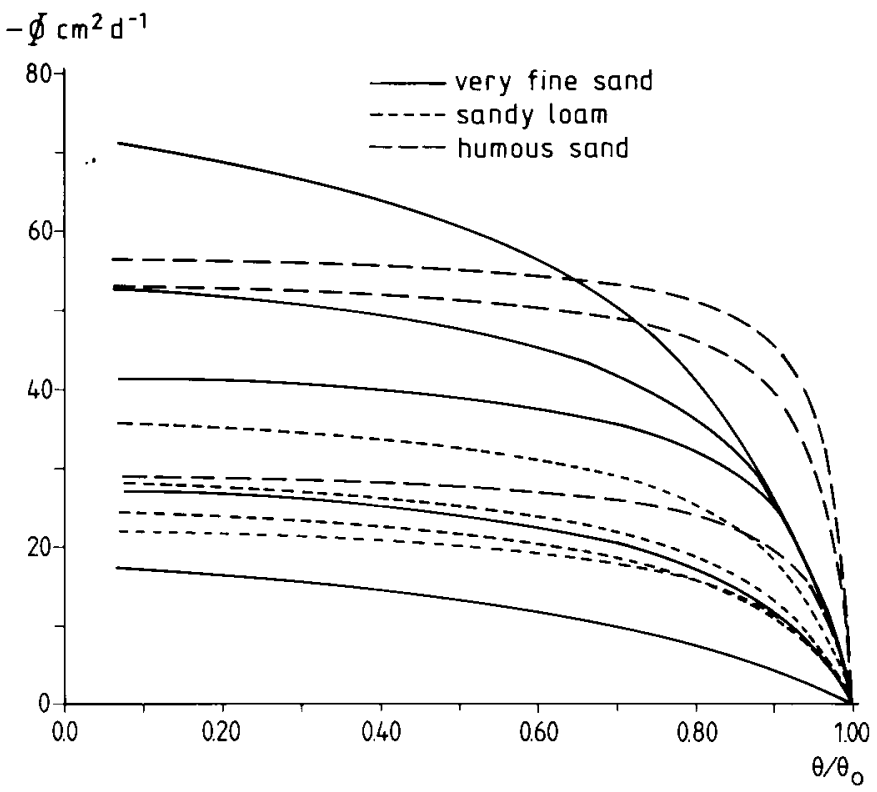

Fig. 3. Matric flux potential curves obtained by the proposed method for three different soil materials. Corresponding dry bulk densities for individual samples of each texture group in $\mathrm{Mg} \mathrm{m}^{-3}$, from top to bottom: very fine sand $0.924,0.911,0.934,0.938,0.923$; sandy loam $1.038,1.049,1.081,1.047$; humous sand $1.552,1.595,1.308$.

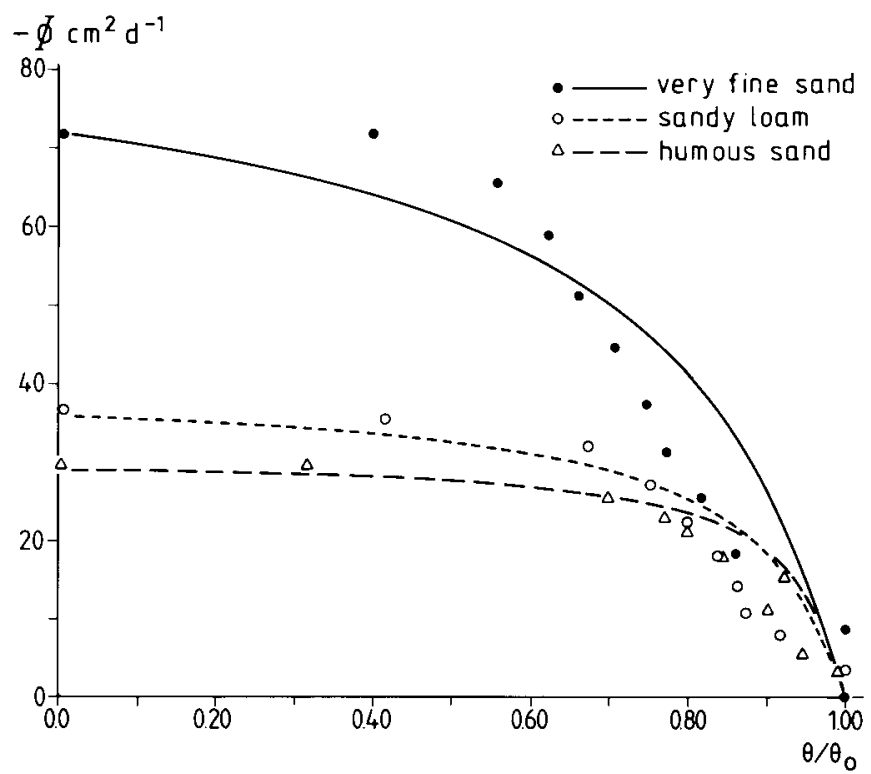

Fig. 4. Matric flux potential curves obtained by the proposed method (lines) compared to $\Phi(\theta)$ based on gravimetric sampling (symbols). 
on the hot air method introduced by Arya et al. (1975).

In some instances a correction on $\Delta W$ had to be applied to account for a non-negligible resistance of the porous plate if $\theta$ in the sampled bottom slice did not equal the initial moisture content.

\section{Evaluation of inherent errors}

Various error sources associated with the application of Eqs. 7 and 11 are to be identified. We can safely state that direct measurement errors in $\Delta W$ and $q_{\max }$ will generally be small as compared to errors arising from failure to meet the assumptions inherent to the method. The relative magnitude of errors in $\Delta W$ and $q_{\max }-$ and hence in $\mathrm{f}(B)$ and $A /(1+B)$, respectively - will be analysed for the cases of (a) a non-negligible gravity term in Eq. 1 , and (b) assessment of $\left(\Delta W, q_{\max }\right)$ under conditions too far from steady state. Furthermore, errors due to the presence of transport mechanisms other than isothermal vapour diffusion and isothermal liquid flow will be discussed briefly.

Errors due to gravity

Taking into account the effect of gravity, the integrated form of Eq. 1 becomes

$$
\left.\int_{0}^{\Phi_{\min }} \mathrm{d} \Phi=-q z\right]_{0}^{L}-\int_{0}^{L} k(z) \mathrm{d} z \quad\left(\mathrm{~L}^{2} \mathrm{~T}^{-1}\right)
$$

with $\Phi_{\min }$ the minimum value that the (negative) matric flux potential can attain. The absolute error in the term $A /(1+B)$ due to gravity is equal to the second term in the right-hand side of Eq. 12. In order to delimit the method's applicability, it is worth trying to quantify this term in relation to the parameters $A, B, L$ and $k_{0}$, the latter being the conductivity at $\theta_{0}$. To this purpose we should seek a link between $k(\theta)$ and $D(\theta)$, which need be approximately correct for high $\theta$ values only, since the sharp decrease of $k$ with $\theta$ renders the drier part of the $k(\theta)$ curve irrelevant to the integral in Eq. 12. Such a link was provided by Philip (1969) and was applied by Talsma \& Parlange (1972) in their paper on infiltration as

$$
\frac{\left(\theta-\theta_{\mathrm{i}}\right) D(\theta)}{\mathrm{d} k / \mathrm{d} \theta} \approx \frac{\int_{\theta_{\mathrm{i}}}^{\theta_{1}}\left(\theta-\theta_{\mathrm{i}}\right) D(\theta) \mathrm{d} \theta}{\int_{\theta_{\mathrm{i}}}^{\theta_{1}}(\mathrm{~d} k / \mathrm{d} \theta) \mathrm{d} \theta}
$$

where the subscript $\mathrm{i}$ in their context referred to the initial status (prior to infiltration) and the subscript 1 to the surface condition after starting infiltration. Later, the relation was used over the full range of $\theta$ at several instances (e.g. Parlange \& Smith, 1976; Smith \& Parlange, 1978; Giraldez \& Sposito, 1985). With these authors we suppose here that the proportionality expressed in Eq. 13 is valid over the full $\theta$ range for soils with a strong dependency of $k$ upon $\theta$. For soils with slowly varying $k(\theta)$ in the wet range, an empirical parameter $\alpha$ is introduced following Par- 
lange et al. (1982) and Giraldez \& Sposito (1985). This parameter then varies between 0 (for constant $k(\theta)$ ) and 1 (for soils behaving according to Eq. 13). On the basis of the above assumptions we may then write the relative hydraulic conductivity as

$$
\frac{k(\theta)}{k_{0}}=1-\alpha \frac{\int_{\theta}^{\theta_{0}} \theta D(\theta) \mathrm{d} \theta}{\int_{0}^{\theta_{0}} \theta D(\theta) \mathrm{d} \theta}
$$

The subscript 0 in Eq. 14 refers to the condition at the lower boundary of the sample. Thus introduction of $\alpha$ gives a generalization of Eq. 13 to cover soils between two extremes. The $D(\theta)$ function required in Eq. 14 is obtained as an expression in $A, B$ and $x$ by differentiation of Eq. 3 , yielding

$$
D(\mathrm{x})=\frac{A B}{\theta_{0}(x+B)^{2}}
$$

Combination of Eq. 14, Eq. 15 and the 'gravity-free' moisture profile (Eq. 9) gives after some manipulation an upper estimate of the gravity error term in Eq. 12, which is here presented as a relative error in $A /(1+B)$ :

$$
\frac{\mathrm{d}(A /(1+B))}{A /(1+B)}=\frac{k_{0} L}{A} \mathbf{F}(\alpha, B)
$$

The function $\mathrm{F}(\alpha, B)$ - see Appendix - may be identified as a scaled relative error; the dimensionless factor $S \equiv k_{0} L / A$ is then interpreted as a scaling coefficient, the 'gravity flux' and the 'matric flux' being linear functions of $k_{0}$ and $A / L$, respectively. This scaled relative error is shown as a function of $B$ in Fig. 5 for selected values of $\alpha$. From the graphs in this figure it can be inferred that a scaling coefficient $S$ of 0.1 reduces the relative gravity error in $A /(1+B)$ to $5-10 \%$ (for high to low $\alpha$ respectively).

The gravity error in $\Delta W$ and hence in $\mathrm{f}(B)$ may be estimated on the basis of $S$ and $\mathrm{F}(\alpha, B)$. Recalling that the weight loss $\Delta W$ was associated with the shape of the moisture profile at stationarity, it follows that in order to take gravity into account the moisture profile in Eq. 9 should be replaced by the profile implied in the exact relation

$$
q z=\frac{A x}{x+B}-\int_{0}^{z} k(z) \mathrm{d} z
$$

If we accept a gross overestimation of the gravity error, Eq. 17 may be simplified to

$$
\frac{A x}{x+B}=\frac{A z}{(1+B) L}+\frac{L-z}{L} \int_{0}^{L} k(z) \mathrm{d} z
$$

which can be combined with Eq. 10 and Eq. 11 to give $\Delta W$, employing $\mathrm{F}(\alpha, B)$ to 


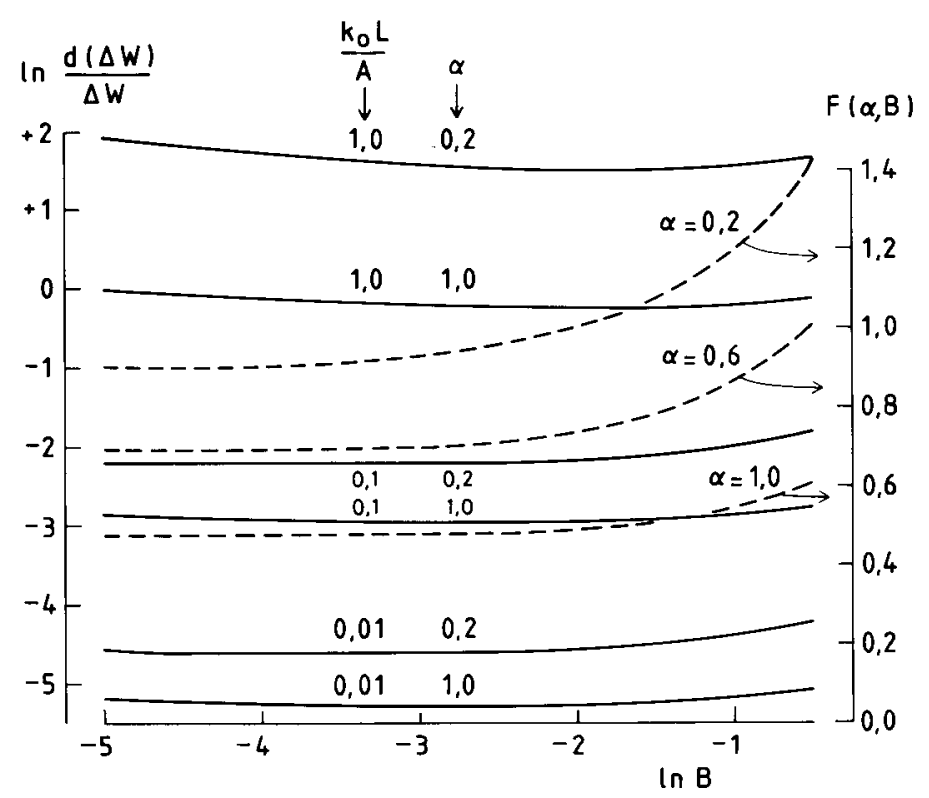

Fig. 5. Left-hand axis and solid lines: relative error in $\mathrm{f}(B)$ as a function of $B$ for various values of the scaling coefficient $k_{0} L / A$ and two values of $\alpha$. Right-hand axis and broken lines: scaled relative error in $A /(1+B)$ for three values of $\alpha$.

express the integral in Eq. 18. The gravity errors thus calculated for $\mathrm{f}(B)$ are also plotted in Fig. 5. It follows from these curves that a scaling coefficient smaller than 0.1 brings the relative error in $\mathrm{f}(B)$ down to below $10 \%$.

In view of the natural variability in soil hydraulic properties, we consider errors up to $10 \%$ in $\mathrm{f}(B)$ and in $A /(1+B)$ as acceptable. To stay below this limit, the scaling coefficient should be adjusted experimentally to a value below 0.1 by choosing suitable values for $L$ and $\theta_{0}$. For standard core rings ( $L=50 \mathrm{~mm}$ ), application of a small negative pressure of -1 to $-5 \mathrm{kPa}$ at the base of the sample is sufficient for most soils to make gravity errors negligible. Analysis of the data by Mualem \& Dagan (1976) mentioned before showed that if $\theta_{0}$ is chosen such that $k\left(\theta_{0}\right)=1 \mathrm{~mm} / \mathrm{d}$, the values of $A$ range between $0.5 \times 10^{-3}$ and $5.0 \times 10^{-3} \mathrm{~m}^{2} \mathrm{~d}^{-1}$. Corresponding $B$ values are then between 0.05 and 0.5 for most soils.

\section{Errors due to non-stationarity}

Measurement under conditions too far from stationarity also causes errors that invalidate Eq. 7 and Eq. 11. Description of the drying process for a soil column of given $B$ under the supposed conditions can be normalized by the introduction of a characteristic parameter $G$ and a scaled time $\tau$, both of which are non-dimensional:

$$
\begin{aligned}
& G \equiv\left(E_{\mathrm{pot}} L\right) / A, \\
& \tau \equiv\left(t E_{\mathrm{pot}}\right) /\left(L \theta_{0}\right)
\end{aligned}
$$




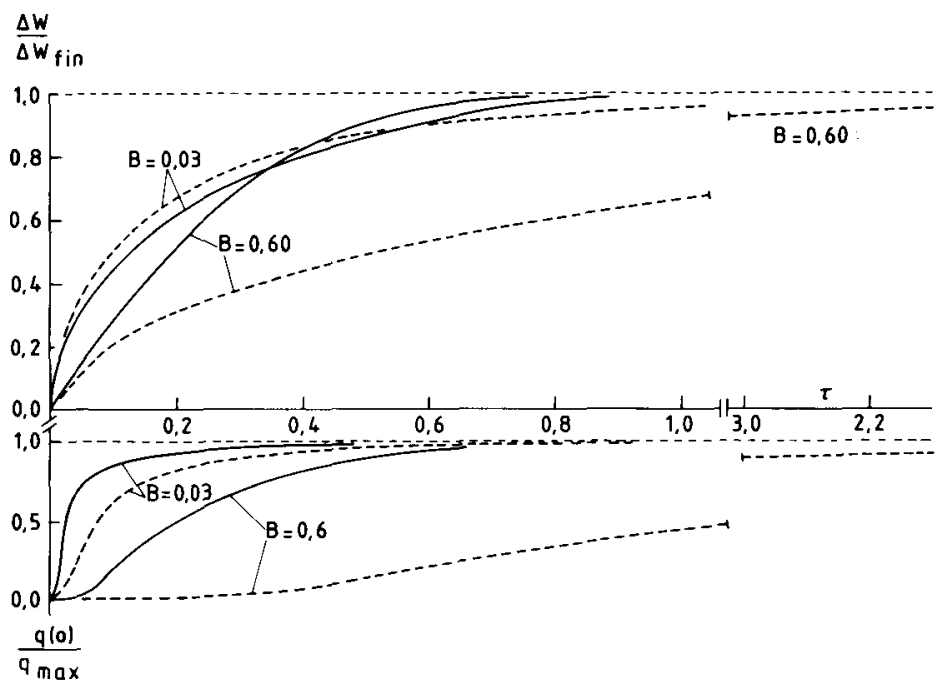

Fig. 6. Relative weight loss $\Delta W$ and relative base flux $q(0)$ versus scaled time for two values of $B$. Solid lines: $G=1$, broken lines: $G=10$.

$E_{\mathrm{pot}}$ in these expressions is the evaporative demand at the soil surface, and $t$ is the time. The course of the two relative errors (as defined below) in scaled time are then unique functions of $G$ and $B$.

In order to estimate errors due to non-stationarity, we may inspect again the behaviour of $\Delta W$ and the base flux $q(0)$ in time. The relative errors in $A /(1+B)$ and $\mathrm{f}(B)$ are $\left(q(0)-q_{\max }\right) / q_{\max }$ and $\left(\Delta W-\Delta W_{\text {fin }}\right) / \Delta W_{\text {fin }}$, respectively, where $\Delta W_{\text {fin }}$ is the final value of $\Delta W$ to be reached at steady state. The course of these errors in time can be read from Fig. 6 where $q(0) / q_{\max }$ and $\Delta W / \Delta W_{\text {fin }}$, obtained by numerical simulation, are plotted versus $\tau$. (To perform the numerical calculations, the fourth-order Runge-Kutta predictor-corrector scheme was used.) The figure shows the results for two extreme values of $B$ and two values of $G$. It is concluded that the time required to reduce the errors in $\mathrm{f}(B)$ and in $A /(1+B)$ to $5-10 \%$ strongly depends on the values of $B$ and $G$. Samples of a well sorted material ('narrow' particle size distribution) can be expected to have a low $B$ value and hence require relatively little time to yield accurate estimates of $A /(1+B)$. Silty materials are supposed to have relatively high $A$ values (so low $G$ ), and therefore would show a $q(0)$ that approaches relatively fast to $q_{\max }$. Clearly it appears from the definitions of $G$ and $\tau$ that a simultaneous increase of $E_{\text {pot }}$ and decrease of $L$ very effectively reduces the actual time $t$ to reach a preset error level. A typical case of $A=5 \times 10^{-3} \mathrm{~m}^{2} \mathrm{~d}^{-1}, B=$ $0.03, E_{\mathrm{pot}}=0.1 \mathrm{~m} \mathrm{~d}^{-1}, \theta_{0}=0.3$ and $L=0.05 \mathrm{~m}$ would require one hour to reach the $5 \%$ error level in $A /(1+B)$ but almost 3 hours to pass the same error level in $\mathrm{f}(B)$.

\section{Errors due to transport other than isothermal diffusion}

The occurrence of transport mechanisms other than isothermal vapour diffusion and isothermal liquid flow is the third error source. Problems may arise due to tem- 
perature gradients, inducing liquid and vapour fluxes not related to moisture content gradients. Van Grinsven et al. (1985) analysed the errors due to thermal liquid and vapour fluxes and to viscosity changes as encountered by the 'hot air method', and concluded that errors are probably small due to various compensation mechanisms. The similarity of our method to the hot air method suggests that their conclusions are also valid in this case.

Vapour transport by forced convection in the top of the sample, however, is likely to be a more severe problem under some circumstances. It may cause the development of a dry layer where $\Phi$ is constant, as indicated in Fig. 2. This would require a correction for $L$ and $\Delta W$ in Eq. 7 and Eq. 11. Forced convection due to air turbulence at the surface depends on soil characteristics and on amplitude and frequency of the pressure fluctuations (Scotter \& Raats, 1969). The data presented by those authors indicate that forced convection in media with particle sizes smaller than 1 $\mathrm{mm}$ is probably negligible. The problem might occur in coarser materials and can be reduced by choosing a proper distance between sample and the source of the air stream employed to enhance evaporation.

\section{Conclusions}

The measurement of matric flux potential on the basis of the assumed two-parameter expression for $\Phi(\theta)$ represents a useful alternative to existing methods of measuring soil water diffusivity. Some disadvantages however must be recognized. The method is slow, being based on steady state flow. Also, the neglect of gravity will usually require the application of a negative pressure at the base of the sample, which implies that only the drier part of the $\Phi(\theta)$ curve is measured. This reduces the method's range of applicability.

Some advantages, on the other hand, make the new method attractive as compared to alternatives. The procedure is very simple and labour extensive: only one flux, initial and final weight, and oven-dry weight of the entire sample are to be measured. The method is non-destructive and can be applied to all sample sizes, since there are no limitations set by slicing, tensiometer installation etc. This renders the procedure especially promising to measurements on thin crusts, thin impeding layers, or on hard or brittle materials. As yet, however, it has not been applied to such samples and still needs to be worked out further. Finally, if one is interested in the integral characteristic $\Phi(\theta)$, direct measurement of this curve supposedly gives much more accurate results than measurement of $D(\theta)$ or $k(\theta)$ and $p(\theta)$ relations followed by mathematical integration. The methods to determine the latter characteristics are usually accurate only over a narrow range of $\theta$.

\section{References}

Arya, L. M., D. Farrell \& G. R. Blake, 1975. A field study of soil water depletion patterns in presence of growing soybean roots. I. Determination of hydraulic properties of the soil. Soil Science Society of America Journal 39: 424-430. 
Berge, H. F. M. ten, 1986. Heat and water transfer at the bare soil surface: aspects affecting thermal imagery. Doctoral thesis, Wageningen Agricultural University, 290 pp.

Gardner, W. R., 1958. Some steady state solutions of the unsaturated moisture flow equation with application to evaporation from a water table. Soil Science 85: 228-232.

Giraldez, J. V. \& G. Sposito, 1985. Infiltration in swelling soils. Water Resources Research 21: 33-44.

Grinsven, J. J. M. van, C. Dirksen \& W. Bouten, 1985. Evaluation of the hot air method for measuring soil water diffusivity. Soil Science Society of America Journal 49: 1093-1099.

Kirchhoff, G., 1894. Vorlesungen über die Theorie der Wärme. Ed. M. Planck, Teubner Verlag, Leipzig, 13 pp.

Klute, A., 1952. A numerical method for solving the flow equation for water in unsaturated materials. Soil Science 73: 105-117.

Knight, J. H. \& J. R. Philip, 1974. Exact solutions in non-linear diffusion. Journal of Engineering Mathematics 8: 219-227.

Mualem, Y. \& G. Dagan, 1976. Development of methods, tools and solutions for unsaturated flow with application to watershed hydrology and other fields. US-Israel Binational Science Foundation, Report Research Project 442. Technion Israel Institute of Technology, Haifa, Israel, 143 pp.

Parlange, J.-Y. \& R. E. Smith, 1976. Ponding time for variable rainfall rates. Canadian Journal of Soil Science 56: 121-123.

Parlange, J.-Y., I. Lisle, R. D. Braddock \& R. E. Smith, 1982. The three-parameter infiltration equation. Soil Science 133: 337-341.

Philip, J. R., 1969. Theory of infiltration. Advances in Hydroscience 5: 215-296.

Philip, J. R., 1971. General theorem on steady state infiltration from surface sources, with application to point and line sources. Soil Science Society of America Proceedings 35: 867-871.

Raats, P. A. C., 1970. Steady infiltration from line sources and furrows. Soil Science Society of America Proceedings 34: 709-714.

Raats, P. A. C. \& W. R. Gardner, 1971. Comparison of empirical relationships between pressure head and hydraulic conductivity and some observations on radially symmetric flow. Water Resources $R e$ search 7: $921-928$.

Raats. P. A. C. 1977. Laterally confined. steady flows of water from sources and sinks in unsaturated soils. Soil Science Society of America Proceedings 41: 294-304.

Scotter, D. R. \& P. A. C. Raats, 1969. Dispersion of water vapour in soil due to air turbulence. Soil Science 108: 170-176.

Shaykewich, C. F. \& L. Stroosnijder, 1977. The concept of matic flux potential applied to simulation of evaporation from soil. Netherlands Journal of Agricultural Science 25: 63-82.

Smith, R. E. \& J. Y. Parlange, 1978. A parameter-efficient hydrologic infiltration model. Water Resources Research 14: 533-538.

Talsma, T. \& J. Y. Parlange, 1972. One-dimensional vertical infiltration. Australian Journal of Soil Research 10: 143-150.

Truesdell, C. \& R. A. Toupin. 1960. The classical field theories. In: S. Flügge (Ed.), Handbuch der Physik, Vol III/1, p. 226-293. Springer-Verlag, Berlin.

Vauclin, M., R. Haverkamp \& G. Vachaud, 1979. Résolution numerique d'une équation de diffusion non-lineaire. Presse Universitaire de Grenoble, France, 183 pp.

Warrick, A. W., 1974. Time-dependent linearized infiltration. I. Point sources. Soil Science Society of America Proceedings 38: 383-386.

\section{Appendix. Derivation of $F(\alpha, B)$}

Substitution of Eq. 15 in Eq. 14 gives upon integration:

$$
k(x)=k_{0}\left(1-\alpha\left[\frac{x-1}{\beta(x+B)}+\frac{1}{\beta B}+\frac{1}{\beta} \ln \left(\frac{B}{x+B}\right)\right]\right)
$$


H. F. M. TEN BERGE, K. METSELAAR AND L. STROOSNIJDER

with $\beta \equiv \ln (B /(1+B))+1 / B$

The slope of the moisture profile follows from Eq. 9 as

$$
\frac{\mathrm{d} x}{\mathrm{~d} z}=\frac{(x+B)^{2}}{B(1+B) L}
$$

The integral in Eq. 12 is written as

$$
\int_{0}^{L} k(z) \mathrm{d} z=\int_{0}^{1} k(x)(\mathrm{d} z / \mathrm{d} x) \mathrm{d} x
$$

and solved by combining Eq. A1 with Eq. A2 and subsequent integration. Division through $A /(1+B)$ then gives Eq. 16 where

$$
\begin{aligned}
\mathrm{F}(\alpha, B) \equiv & \left(\frac{1+B}{\beta}\right) \times \\
& {\left[-\gamma^{\prime}-\alpha B \ln (1+B)+\gamma^{\prime}\left(\frac{B+1}{B}\right)+\alpha(1+B) \ln B+\frac{\alpha(1+B)}{2 B}\right] }
\end{aligned}
$$

and

$$
\gamma^{\prime} \equiv \beta B-\alpha(1+B \ln B-B / 2)
$$

